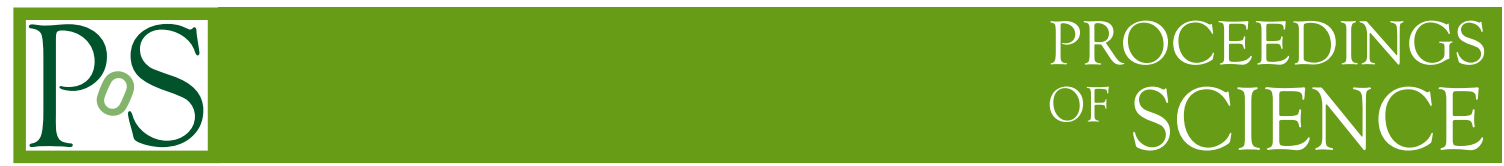

\title{
Doubly-charged Higgs searches by ATLAS
}

\author{
Francesco Nuti*, on behalf of the ATLAS Collaboration \\ University of Melbourne, Australia \\ E-mail: francesco.nutiecern.ch
}

A search for new physics in dilepton final states with same electric charge, $e^{ \pm} e^{ \pm}, e^{ \pm} \mu^{ \pm}$and $\mu^{ \pm} \mu^{ \pm}$, with the ATLAS detector at LHC, is presented. No significant contribution from beyond the Standard Model physics is observed in $4.7 \mathrm{fb}^{-1}$ of $\sqrt{s}=7 \mathrm{TeV}$ proton-proton collisions. An upper limit at 95\% Confidence Level (CL) on the fiducial cross section for new physics processes is derived, ranging between $1.7 \mathrm{fb}$ and $64 \mathrm{fb}$ depending on the dilepton invariant mass and the lepton flavour combination. A 95\% CL limit is also set on the cross section for pair production of doubly-charged Higgs as a function of its mass, varying between $25 \mathrm{fb}$ and $0.6 \mathrm{fb}$. Lower limits on the mass of doubly-charged Higgs bosons in the Left-Right symmetric models and in the Zee-Babu models are obtained.

Prospects for Charged Higgs Discovery at Colliders - CHARGED 2014,

16-18 September 2014

Uppsala University, Sweden

\footnotetext{
*Speaker.
} 


\section{Introduction}

Same-sign leptons are a very clear signature of new physics. The production of same electric charge (same-sign) lepton pairs in proton-proton (pp) collisions occurs rarely in Standard Model (SM) processes. However, in Supersymmetry [1], universal extra dimensions [2], Left-Right symmetric models [3, 4, 5] and Zee-Babu models [6, 7, 8], processes with same-sign lepton pairs have large cross section. Left-Right symmetric models and Zee-Babu models predict doubly-charged Higgs bosons (DCH) that can decay into same-sign leptons. They would be observed as resonances in the dilepton invariant mass spectrum. The discovery of a DCH would have double importance since it may provide hints about the mechanism of neutrino masses generation. Neutrinos may acquire mass via the interaction with doubly-charged fields $[8,9,10]$. The search for same-sign leptons with the ATLAS experiment [11] at the LHC has therefore the potential to observe or constrain several beyond SM processes, DCH production in particular.

This paper describes the analysis of same-sign dilepton final states performed in $\sqrt{s}=7 \mathrm{TeV}$ pp collisions and reconstructed with the ATLAS detector, using an integrated luminosity of $4.7 \mathrm{fb}^{-1}$ with an uncertainty of $3.9 \%$. The same-sign dilepton pairs can be two electrons $\left(e^{ \pm} e^{ \pm}\right)$, two muons $\left(\mu^{ \pm} \mu^{ \pm}\right)$or one electron and one muon $\left(e^{ \pm} \mu^{ \pm}\right)$. The leptons must come from the same vertex of interaction and must have large transverse momentum $\left(p_{\mathrm{T}}\right)$. They must satisfy isolation and identification criteria as described in Section 2. The resulting dilepton invariant mass distributions are examined and compared with the SM prediction obtained according to Section 3. No significant excess is found and exclusion limits are set on the fiducial cross section of new physics (Section 5). A similar search has been performed by the CDF experiment [12] and has not observed any evidence for new physics. Exclusion limits on the pair production of DCH and on the mass of DCH for Left-Right symmetric models and Zee-Babu models are discussed in section 6. More details about the analysis can be found in $[13,14]$. The CMS experiment search for DCH in $\sqrt{s}=7 \mathrm{TeV}$ data has led to similar results [15].

\section{Event Selection}

Data were collected using single-muon and single-electron triggers with at least one muon with $p_{\mathrm{T}}>20 \mathrm{GeV}$ or one electron with $p_{\mathrm{T}}>25 \mathrm{GeV}$, associated to the trigger. This ensures to work on the plateau of efficiency for lepton triggers. Any other lepton in the event must have $p_{\mathrm{T}}>20 \mathrm{GeV}$. The analysis makes use of muons and electrons selected with high quality criteria optimized to reject leptons from hadrons decays and from interactions with the detector material.

The electron and muon reconstruction is described in [16, 17]. Tight identification criteria based on shower shapes in the calorimeters, track qualities and matching are applied. Leptons from heavy-flavour decays are reduced by requiring small impact parameter of the lepton track from the primary vertex. The primary vertex is defined as the vertex which has the highest squared- $p_{\mathrm{T}}$ sum of associated tracks found in the event. To further suppress leptons produced in jets or jets misidentified as leptons, the leptons are required to be isolated. The selection is based on the sum of the $p_{\mathrm{T}}$ of all tracks in a cone of size $R=\sqrt{(\Delta \eta)^{2}+(\Delta \phi)^{2}}=0.3(0.4)$ surrounding the electron (muon) track. For electrons, a further isolation requirement based on the transverse energies in the electromagnetic and hadronic calorimeter cells around the electron direction in a cone of size $R=0.2$ is also 
applied. In addition leptons in a cone of size $R=0.4$ around jets with $p_{\mathrm{T}}>25 \mathrm{GeV}+p_{\mathrm{T}}^{\ell} \times 0.05$, where $p_{\mathrm{T}}^{\ell}$ is the lepton $p_{\mathrm{T}}$ are rejected. The jets in this analysis are reconstructed from the energy deposits in the calorimeter using the anti- $k_{t}$ algorithm [18] with a radius parameter of 0.4.

Events are selected if they contain at least two leptons $\left(e^{ \pm} e^{ \pm}, \mu^{ \pm} \mu^{ \pm}, e^{ \pm} \mu^{ \pm}\right)$of the same electric charge satisfying the above criteria and with an invariant mass larger than $15 \mathrm{GeV}$ to avoid hadronic resonances as $J / \psi$. In addition, in the $e^{ \pm} e^{ \pm}$channel, the mass range $70<m_{\ell \ell}<$ $110 \mathrm{GeV}$ (the $Z$ peak in the same-sign region) is removed and is used for charge misidentification studies.

\section{Background estimation}

Monte Carlo (MC) simulations are used to estimate the backgrounds with leptons produced by $W, Z$ or $\tau$ decays (prompt) and to model hypothetical signal events. A Data-Driven technique is adopted to provide an estimate of backgrounds where at least one lepton is produced in a hadronic decay or where the hadron is misidentified as lepton.

The main irreducible backgrounds in this analysis are the purely leptonic decays of $W Z$ and $Z Z$ processes. A small contribution arises from $W^{ \pm} W^{ \pm}$production in association with at least two jets and from the production of a $W$ or $Z$ boson in association with a top-quark pair ( $t \bar{t} W$ and $t \bar{t} Z$ ).

Opposite-sign lepton pairs where the charge for one of the leptons is wrongly reconstructed is a reducible background. It is due to either wrong track association, or wrong track curvature measurement or reconstruction of the opposite-sign lepton of a trident event, the main occurrence. In a trident event, the lepton radiates an high-momentum photon which converts into an $e^{+} e^{-}$pair. Charge misidentification is found to be relevant for electrons and negligible for muons. Backgrounds with charge misidentified leptons are Drell-Yan $\left(Z / \gamma^{*}+\right.$ jets $), t \bar{t}, W^{+} W^{-}$and $W t$, and they are derived from simulations. The misidentification probability is corrected in MC to reproduce data. The processes $W \gamma$ and $Z \gamma$, where the photon converts to an $e^{+} e^{-}$pair, are also accounted for.

Same-sign dilepton signals from several beyond SM processes are simulated to evaluate the efficiency and the acceptance used in the limits. The processes are chosen to cover a broad range of event topologies. They are: pair production of doubly-charged Higgs bosons via a virtual $Z / \gamma^{*}$ exchange [19]; right-handed $W$ bosons $\left(W_{\mathrm{R}}\right)$ decaying to a charged lepton and a right-handed neutrino $\left(N_{\mathrm{R}}\right)$ [20]; production of a fourth-generation heavy $b^{\prime} \bar{b}^{\prime}$ pair with the $b^{\prime}$ quarks decaying into $W t$ [21]; like-sign top-quark pair production via a t-channel exchange of a $Z^{\prime}$ [22].

The cross sections for simulated backgrounds events are normalised to next-to-leading order (NLO) or to next-to-next-to-leading order (NNLO) QCD. Signal cross sections are calculated at LO QCD. The simulated background and signal samples are processed using the GEANT4-based ATLAS detector simulation package [23]. Multiple inelastic pp interactions are overlaid on the hard-scatter events to emulate the multiple pp interactions ('pile-up') occurring in the bunch crossings. Simulations are corrected for differences in pile-up conditions, particle selection efficiencies, momentum scales and momentum resolution between data and simulation.

Non-prompt leptons come mainly from semi-leptonic decays of heavy flavour hadrons ( $b$ and $c$ ) and from decays of long-lived particles. Hadrons misidentified as leptons are also considered non-prompt leptons. The main processes contributing to the non-prompt background are $W+$ jets, $Z / \gamma^{*}+$ jets, $t \bar{t}$, multi-jet QCD. Their contribution in the signal region is obtained from data. For 
both electrons and muons, the non-prompt background determination relies on measuring a transfer factor $f$ that relates the number of non-prompt leptons failing one or more of the analysis selection requirements ${ }^{1}\left(N_{\mathrm{F}}\right)$ and the number of those passing the whole selection $\left(N_{\mathrm{P}}\right)$. The transfer factor $f$ is measured as a function of lepton $p_{\mathrm{T}}$ and $|\eta|$ in control regions enriched of non-prompt leptons after the subtraction prompt contaminations from data:

$$
f\left(p_{\mathrm{T}},|\eta|\right)=\frac{N_{\mathrm{P}}\left(p_{\mathrm{T}}\right)}{N_{\mathrm{F}}\left(p_{\mathrm{T}}\right)} \times \frac{N_{\mathrm{P}}(|\eta|)}{N_{\mathrm{F}}(|\eta|)} \times\left\langle\frac{N_{\mathrm{P}}(|\eta|)}{N_{\mathrm{F}}(|\eta|)}\right\rangle^{-1}
$$

The total number of pairs with non-prompt leptons in the signal region, $N_{\mathrm{NP}}$, is predicted by summing reweighted pairs with leptons failing the analysis selection:

$$
N_{\mathrm{NP}}=\sum_{i=1}^{\mathrm{N}_{\mathrm{P}+\mathrm{F}}} f\left(p_{\mathrm{T} i},\left|\eta_{i}\right|\right)-\sum_{i=1}^{\mathrm{N}_{\mathrm{F}+\mathrm{F}}} f\left(p_{\mathrm{T} i}^{\prime},\left|\eta_{i}^{\prime}\right|\right) \times f\left(p_{\mathrm{T} i}^{\prime \prime},\left|\eta_{i}^{\prime \prime}\right|\right)
$$

$N_{\mathrm{P}+\mathrm{F}}\left(N_{\mathrm{F}+\mathrm{F}}\right)$ is the number of pairs with one (two) lepton(s) failing the selection. The transfer factors $f$ are function of the $p_{\mathrm{T}}$, the $\eta$ and the flavour of the leptons of the $i$-th pair which fail the selection. The last term in the equation avoids double countings and the indices ' and " are used to distinguish between the two leptons failing the selection in the pair.

The predictions for prompt, non-prompt and charge misidentification backgrounds have been validated in dedicated control regions.

\section{Systematic uncertainties}

The systematic uncertainties on the trigger selection, particle reconstruction, identification, energy scale and resolution, the luminosity, MC statistics, data-driven corrections, theoretical cross sections are taken into account. The main uncertainty on the number of predicted same-sign pairs is due to the non-prompt estimation method.

\section{Fiducial cross section results}

Tabel 1 shows the number of observed and expected pairs in the $e^{ \pm} e^{ \pm}, e^{ \pm} \mu^{ \pm}$and $\mu^{ \pm} \mu^{ \pm}$ signal regions. Good agreement between data and predictions is observed in all channels. The

\begin{tabular}{l|c|c|c|c|c}
\hline & \multicolumn{5}{|c}{ Number pairs } \\
\hline & Prompt & Non-Prompt & $e^{ \pm}$charge misid. & Total bkg & Data \\
\hline$e^{ \pm} e^{ \pm}$ & $101 \pm 13$ & $75 \pm 21$ & $170 \pm 33$ & $346 \pm 44$ & 329 \\
$e^{ \pm} \mu^{ \pm}$ & $346 \pm 43$ & $151 \pm 47$ & $142 \pm 28$ & $639 \pm 71$ & 658 \\
$\mu^{ \pm} \mu^{ \pm}$ & $205 \pm 26$ & $42 \pm 14$ & $0_{-0.0}^{+4.9}$ & $247_{-29}^{+30}$ & 264 \\
\hline
\end{tabular}

Table 1: Expected and observed numbers of same-sign lepton pairs in the $e^{ \pm} e^{ \pm}, e^{ \pm} \mu^{ \pm}$and $\mu^{ \pm} \mu^{ \pm}$channels. Systematic uncertainties are shown [13].

largest upward deviation in the same-sign dilepton invariant mass spectrum occurs about $8 \%$ of

\footnotetext{
${ }^{1}$ In this analysis the lepton fails the isolation criteria but satisfies the other analysis requirements.
} 
the time in background-only pseudo-experiments. The Confidence Level $\left(\mathrm{CL}_{\mathrm{S}}\right)$ method [24] is used to compute $95 \%$ upper limits on the number of same-sign lepton pairs $\left(N_{95}\right)$ from physics beyond the SM. The $N_{95}$ limit can be translated into an upper limit on the fiducial cross section $\sigma_{95}^{\mathrm{fid}}=N_{95} /\left(\varepsilon_{\mathrm{fid}} \times \int L \mathrm{~d} t\right)$, where $\varepsilon_{\mathrm{fid}}$ is the fiducial efficiency, and $\int L \mathrm{~d} t$ is the integrated luminosity. The fiducial efficiency is the ratio of the number of reconstructed lepton pairs to the number of true lepton pairs satisfying the fiducial selection. The fiducial selection is optimized to reproduce at particle level, the selections applied at reconstruction level. This definition minimises the dependence of the $\varepsilon_{\text {fid }}$ from the signal model. The lowest fiducial efficiency among all the signal processes considered is used in the $\sigma_{95}^{\text {fid }}$ calculation to obtain results that do not depend on the signal model. The 95\% CL upper limits on the fiducial cross section are shown in Figure 1 separately for each flavour combination and for various invariant mass thresholds. For all final states the observed limits are

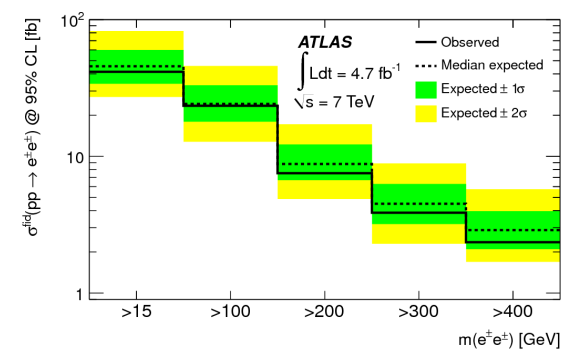

(a)

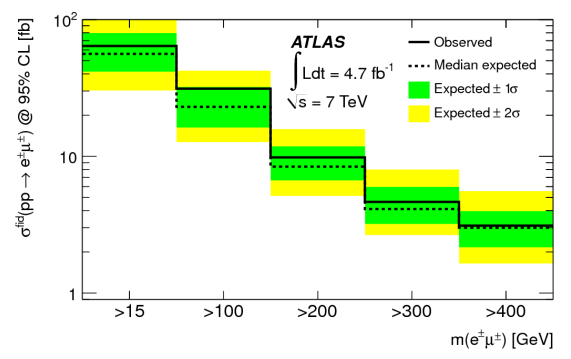

(b)

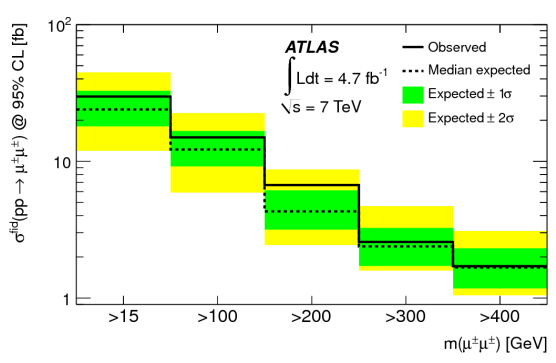

(c)

Figure 1: Fiducial cross section limits for new physics at 95\% CL for (a) $e^{ \pm} e^{ \pm}$, (b) $e^{ \pm} \mu^{ \pm}$and (c) $\mu^{ \pm} \mu^{ \pm}$pairs, as a function of the lower bound on the lepton pair mass. The green and yellow bands show the $1 \sigma$ and $2 \sigma$ bands on the expected limits [13].

generally within $1 \sigma$ of the expected limits, which are obtained using pseudo-experiments in the background only hypothesis. Observed limits vary between $1.7 \mathrm{fb}$ and $64 \mathrm{fb}$.

\section{Doubly-charged Higgs limits}

A special treatment is made for the production of doubly-charged Higgs pairs. Limits on the production cross section times branching ratio $(B R)$ of the decay into a specific lepton pair $\left(H^{ \pm \pm} \rightarrow\right.$ $\left.\ell^{ \pm} \ell^{\prime \pm}\right)$ are set as a function of the DCH mass. The cross section times $B R$ is determined using $\sigma_{H H} \times B R=N_{H}^{\mathrm{rec}} /\left(2 \times A \times \varepsilon \times \int L \mathrm{~d} t\right)$, where $N_{H}^{\mathrm{rec}}$ is the number of reconstructed Higgs boson candidates, $A \times \varepsilon$ is the acceptance times efficiency to find a lepton pair from the $H^{ \pm \pm}$decay, and 
the factor of two accounts for the two same-sign lepton pairs from the DCH bosons in the event. The $A \times \varepsilon$ is calculated as a function of the simulated DCH mass. The dilepton invariant mass spectrum is scanned in mass windows optimized according to the mass resolution of the DCH. Limits on the cross section for pair production of $\mathrm{H}^{++} \mathrm{H}^{--}$bosons times $B R$ are extracted using the $\mathrm{CL}_{S}$ technique. Good agreement is seen between expected and observed limits for each lepton flavour combination. The observed limits range from $25 \mathrm{fb}$ for low masses $\left(m_{H^{ \pm \pm}}<100 \mathrm{GeV}\right)$ to $0.6 \mathrm{fb}$ for high masses $\left(m_{H^{ \pm \pm}}>550 \mathrm{GeV}\right)$.

The results can be translated in lower limits on the mass of left-handed Higgs, $H_{\mathrm{L}}^{ \pm \pm}$, and right-handed Higgs, $H_{\mathrm{R}}^{ \pm \pm}$, for the Left-Right symmetric models. Limits on the mass of the $H_{\mathrm{ZBM}}^{ \pm \pm}$ boson predicted in the Zee-Babu models are also set. The $H_{\mathrm{ZBM}}^{ \pm \pm}$has the same kinematic properties and production rate of the $H_{\mathrm{L}}^{ \pm \pm}$, therefore the same limit. The $H_{\mathrm{R}}^{ \pm \pm}$has same kinematic of the $H_{\mathrm{L}}^{ \pm \pm}$ but a smaller production rate. The mass limits as a function of the branching ratio for $H_{\mathrm{L}}^{ \pm \pm}$and $H_{\mathrm{R}}^{ \pm \pm}$in the three final states are shown in Figure 2. For $B R=1$, the $H_{\mathrm{L}}^{ \pm \pm}$mass and the $H_{\mathrm{ZBM}}^{ \pm \pm}$mass are excluded below $409 \mathrm{GeV}, 375 \mathrm{GeV}$ and $398 \mathrm{GeV}$ while the $H_{\mathrm{R}}^{ \pm \pm}$mass is excluded below 322 $\mathrm{GeV}, 310 \mathrm{GeV}$ and $306 \mathrm{GeV}$ in the $e^{ \pm} e^{ \pm}, e^{ \pm} \mu^{ \pm}, \mu^{ \pm} \mu^{ \pm}$channel respectively.

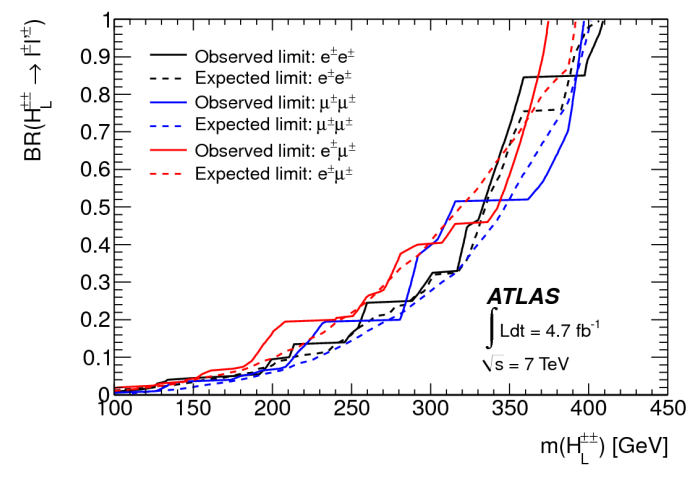

(a)

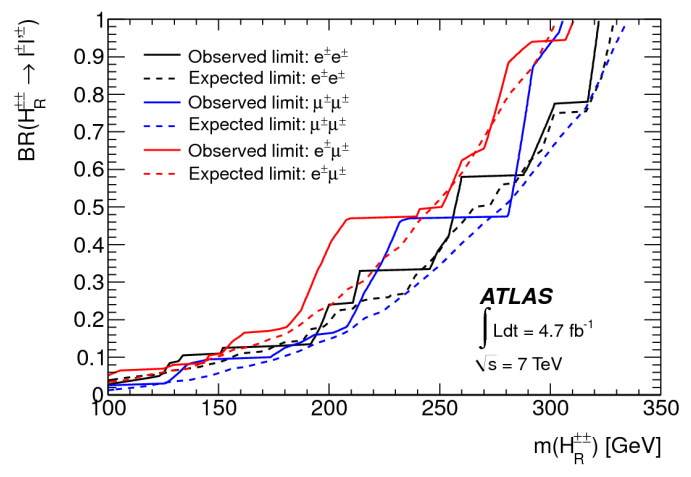

(b)

Figure 2: Observed (solid lines) and expected (dashed lines) 95\% CL limits on $H_{\mathrm{L}}^{ \pm \pm} \rightarrow \ell^{ \pm} \ell^{ \pm}$(a) and $H_{\mathrm{R}}^{ \pm \pm} \rightarrow \ell^{ \pm} \ell^{ \pm}$production (b) in the branching ratio versus $H^{ \pm \pm}$mass plane for the $e^{ \pm} e^{ \pm}, e^{ \pm} \mu^{ \pm}$and $\mu^{ \pm} \mu^{ \pm}$ channel [14].

\section{Summary}

A search for new physics in same-sign $e^{ \pm} e^{ \pm}, e^{ \pm} \mu^{ \pm}$and $\mu^{ \pm} \mu^{ \pm}$pairs with $4.7 \mathrm{fb}^{-1}$ of $\sqrt{s}=7$ $\mathrm{TeV} p p$ collision is presented. No significant deviations from SM are observed and fiducial cross section limits of beyond SM physics with same-sign isolated leptons in the final state are set. The observed limits vary between $1.7 \mathrm{fb}$ and $64 \mathrm{fb}$ as functions of the dilepton invariant mass and flavour combination. Limits on the production of doubly-charged Higgs pairs have been obtained and range from $25 \mathrm{fb}$ to $0.6 \mathrm{fb}$ depending on the DCH mass. For the $H_{\mathrm{L}}^{ \pm \pm}$, the $H_{\mathrm{R}}^{ \pm \pm}$in the Left-Right symmetric models and for the $H_{\mathrm{ZBM}}^{ \pm \pm}$in the Zee-Babu models, lower limits on the boson mass are also derived. 


\section{References}

[1] R. M. Barnett, J. F. Gunion, and H. E. Haber, Discovering supersymmetry with like sign dileptons, Phys. Lett. B 315 (1993) 349, [hep-ph/9306204].

[2] J. Alwall, P. Schuster, and N. Toro, Simplified Models for a First Characterization of New Physics at the LHC, Phys. Rev. D 79 (2009) 075020, [arXiv: 0810.3921 ].

[3] J. C. Pati and A. Salam, Lepton number as the fourth "color", Phys. Rev. D 10 (1974) 275.

[4] R. N. Mohapatra and J. C. Pati, Left-right gauge symmetry and an "isoconjugate" model of CP violation, Phys. Rev. D 11 (1975) 566.

[5] G. Senjanovic and R. N. Mohapatra, Exact left-right symmetry and spontaneous violation of parity, Phys. Rev. D 12 (1975) 1502.

[6] A. Zee, Quantum Numbers of Majorana Neutrino Masses, Nucl. Phys. B 264 (1986) 99.

[7] K. S. Babu, Model of 'Calculable’ Majorana Neutrino Masses, Phys. Lett. B 203 (1988) 132.

[8] M. Nebot, J. F. Oliver, D. Palao, and A. Santamaria, Prospects for the Zee-Babu model at the CERN LHC and low energy experiments, Phys. Rev. D 77 (2008) 093013, [arXiv: 0711.0483 ].

[9] M. Magg and C. Wetterich, Neutrino mass problem and gauge hierarchy, Phys. Lett. B 94 (1980) 61.

[10] T. P. Cheng and L. F. Li, Neutrino masses, mixings, and oscillations in $S U(2) \tilde{A} U U^{\circ}(1)$ models of electroweak interactions, Phys. Rev. D 22 (1980) 2860.

[11] ATLAS Collaboration, The ATLAS Experiment at the CERN Large Hadron Collider, JINST 3 (2008) S08003.

[12] CDF Collaboration, A. Abulencia et al., Inclusive Search for New Physics with Like-Sign Dilepton Events in ppbar Collisions at $\sqrt{s}=1.96$ TeV, Phys. Rev. Lett. 98 (2007) 221803, [hep-ex/0702051].

[13] ATLAS Collaboration, Search for anomalous production of prompt like-sign lepton pairs at $\sqrt{s}=7 \mathrm{TeV}$ with the ATLAS detector, JHEP 12 (2012) 007, [arXiv:1210.4538].

[14] ATLAS Collaboration, Search for doubly-charged Higgs bosons in like-sign dilepton final states at $\sqrt{s}=7$ TeVwith the ATLAS detector, Eur. Phys. J. C 72 (2012) 2244, [arXiv: 1210.5070 ].

[15] CMS Collaboration, A search for a doubly-charged Higgs boson in pp collisions at $\sqrt{s}=7 \mathrm{TeV}$, Eur. Phys. J. C 72 (2012) 2189, [arXiv:1207.2666].

[16] ATLAS Collaboration, Electron reconstruction and identification efficiency measurements with the ATLAS detector using the 2011 LHC proton-proton collision data, Eur. Phys. J. C 74 (2014) 2941, [arXiv:1404.2240].

[17] ATLAS Collaboration, Muon reconstruction efficiency and momentum resolution of the ATLAS experiment in proton-proton collisions at $\sqrt{s}=7 \mathrm{TeV}$ in 2010, to be submitted (2014) [arXiv:1404.4562].

[18] M. Cacciari, G. P. Salam, and G. Soyez, The anti-kt jet clustering algorithm, JHEP 04 (2008) 063, [arXiv:0802.1189].

[19] K. Huitu, J. Maalampi, A. Pietilä and M. Raidal, Doubly charged Higgs at LHC, Nucl. Phys. B 487 (1997) 27, [hep-ph/9606311].

[20] G. Barenboim, Electroweak precision data and right-handed gauge bosons, Eur. Phys. J. C 1 (1998) 369. 
[21] S. Bar-Shalom, M. Geller, S. Nandi, and A. Soni, Two Higgs doublets, a 4th generation and a 125 GeV Higgs: a review, Adv. High Energy Phys. 2013 (2013) 672972, [arXiv: 1208 . 3195 ].

[22] J. A. Aguilar-Saavedra, Effective four-fermion operators in top physics: a roadmap, Nucl.Phys. B 843 (2011) 638; erratum ibid. B851 (2011) 443, [arXiv: 1008 .3562].

[23] ATLAS Collaboration, The ATLAS Simulation Infrastructure, Eur. Phys. J. C 70 (2010) 823, [arXiv:1005.4568].

[24] A. L. Read, Presentation of search results: The CL(s) technique, J. Phys. G 28 (2002) 2693. 